\title{
The GRAVITY young stellar object survey
}

\section{First spatially resolved observations of the $\mathrm{CO}$ bandhead emission in a high-mass YSO}

GRAVITY Collaboration ${ }^{\star}$ : A. Caratti o Garatti ${ }^{1,2,3}$, R. Fedriani ${ }^{1,3,4}$, R. Garcia Lopez ${ }^{1,2,3}$, M. Koutoulaki ${ }^{1,3,5}$, K. Perraut ${ }^{6}$, H. Linz ${ }^{2}$, W. Brandner ${ }^{2}$, P. Garcia ${ }^{7,8,9}$, L. Klarmann ${ }^{2}$, T. Henning ${ }^{2}$, L. Labadie ${ }^{10}$, J. Sanchez-Bermudez ${ }^{2,11}$, B. Lazareff ${ }^{6}$, E. F. van Dishoeck ${ }^{12,14}$, P. Caselli ${ }^{12}$, P. T. de Zeeuw ${ }^{12,14}$, A. Bik ${ }^{13}$, M. Benisty ${ }^{6,9}$, C. Dougados ${ }^{6}$, T. P. Ray ${ }^{1}$, A. Amorim ${ }^{8}$, J.-P. Berger ${ }^{6}$, Y. Clénet $^{15}$,

V. Coudé du Foresto ${ }^{15}$, G. Duvert ${ }^{6}$, A. Eckart ${ }^{10}$, F. Eisenhauer ${ }^{12}$, F. Gao ${ }^{12}$, E. Gendron ${ }^{15}$, R. Genzel ${ }^{12,16}$, S. Gillessen ${ }^{12}$, P. Gordo ${ }^{8}$, L. Jocou ${ }^{6}$, M. Horrobin ${ }^{10}$, P. Kervella' ${ }^{15}$, S. Lacourr ${ }^{15}$, J.-B. Le Bouquin ${ }^{6}$, P. Léna ${ }^{15}$, R. Grellmann ${ }^{10}$, T. Ott ${ }^{12}$, T. Paumard ${ }^{15}$, G. Perrin ${ }^{15}$, G. Rousset ${ }^{15}$, S. Scheithauer ${ }^{2}$, J. Shangguan ${ }^{12}$, J. Stadler ${ }^{12}$, O. Straub ${ }^{12}$, C. Straubmeier ${ }^{10}$, E. Sturm ${ }^{12}$, W. F. Thi ${ }^{12}$, F. H. Vincent ${ }^{15}$, and F. Widmann ${ }^{12}$

1 Dublin Institute for Advanced Studies, 31 Fitzwilliam Place, D02 XF86 Dublin, Ireland e-mail: alessio@cp.dias.ie

2 Max Planck Institute for Astronomy, Königstuhl 17, 69117 Heidelberg, Germany

3 University College Dublin, School of Physics, Belfield, Dublin 4, Ireland

4 Department of Space, Earth \& Environment, Chalmers University of Technology, 41293 Gothenburg, Sweden

5 European Southern Observatory, Karl-Schwarzschild-Str. 2, 85748 Garching, Germany

${ }^{6}$ Univ. Grenoble Alpes, CNRS, IPAG, 38000 Grenoble, France

7 Universidade do Porto - Faculdade de Engenharia, Rua Dr. Roberto Frias, 4200-465 Porto, Portugal

8 CENTRA, Instituto Superior Tecnico, Av. Rovisco Pais, 1049-001 Lisboa, Portugal

9 European Southern Observatory, 19001 Casilla, Santiago 19, Chile

10 I. Physikalisches Institut, Universität zu Köln, Zülpicher Str. 77, 50937 Köln, Germany

11 Instituto de Astronomía, Universidad Nacional Autónoma de México, Apdo. Postal 70264, 04510 Ciudad de México, Mexico

12 Max Planck Institute for Extraterrestrial Physics, Giessenbachstrasse, 85741 Garching bei München, Germany

13 Department of Astronomy, Stockholm University, Oskar Klein Center, 10691 Stockholm, Sweden

14 Leiden Observatory, Leiden University, PO Box 9513, 2300 RA Leiden, The Netherlands

15 LESIA, Observatoire de Paris, PSL Research University, CNRS, Sorbonne Universités, UPMC Univ. Paris 06, Univ. Paris Diderot, Sorbonne Paris Cité, France

16 Department of Physics, Le Conte Hall, University of California, Berkeley, CA 94720, USA

Received 26 January 2020 / Accepted 9 March 2020

\section{ABSTRACT}

\begin{abstract}
Context. The inner regions of the discs of high-mass young stellar objects (HMYSOs) are still poorly known due to the small angular scales and the high visual extinction involved.

Aims. We deploy near-infrared spectro-interferometry to probe the inner gaseous disc in HMYSOs and investigate the origin and physical characteristics of the CO bandhead emission $(2.3-2.4 \mu \mathrm{m})$.

Methods. We present the first GRAVITY/VLTI observations at high spectral $(\mathcal{R}=4000)$ and spatial (mas) resolution of the CO overtone transitions in NGC 2024 IRS 2.

Results. The continuum emission is resolved in all baselines and is slightly asymmetric, displaying small closure phases $\left(\leq 8^{\circ}\right)$. Our best ellipsoid model provides a disc inclination of $34^{\circ} \pm 1^{\circ}$, a disc major axis position angle (PA) of $166^{\circ} \pm 1^{\circ}$, and a disc diameter of $3.99 \pm 0.09$ mas (or $1.69 \pm 0.04 \mathrm{au}$, at a distance of $423 \mathrm{pc}$ ). The small closure phase signals in the continuum are modelled with a skewed rim, originating from a pure inclination effect. For the first time, our observations spatially and spectrally resolve the first four $\mathrm{CO}$ bandheads. Changes in visibility, as well as differential and closure phases across the bandheads are detected. Both the size and geometry of the CO-emitting region are determined by fitting a bidimensional Gaussian to the continuum-compensated CO bandhead visibilities. The CO-emitting region has a diameter of $2.74 \pm_{0.07}^{0.08}$ mas $(1.16 \pm 0.03 \mathrm{au})$, and is located in the inner gaseous disc, well within the dusty rim, with inclination and PA matching the dusty disc geometry, which indicates that both dusty and gaseous discs are coplanar. Physical and dynamical gas conditions are inferred by modelling the $\mathrm{CO}$ spectrum. Finally, we derive a direct measurement of the stellar mass of $M_{*} \sim 14.7_{-3.6}^{+2} M_{\odot}$ by combining our interferometric and spectral modelling results.
\end{abstract}

Key words. stars: formation - stars: massive - infrared: stars - techniques: interferometric - techniques: spectroscopic methods: observational

^ GRAVITY is developed in a collaboration by the Max Planck Institute for Extraterrestrial Physics, LESIA of Paris Observatory and IPAG of Université Grenoble Alpes / CNRS, the Max Planck Institute for Astronomy, the University of Cologne, the Centro Multidisciplinar de Astrofisica Lisbon and Porto, and the European Southern Observatory. 


\section{Introduction}

Accretion discs around high-mass young stellar objects $(M>$ $8 M_{\odot} ; \mathrm{O}$ and early $\mathrm{B}$ spectral types) are key for understanding how massive stars form. However, their structure and main physical properties are poorly known (see Beltrán \& de Wit 2016, and references therein). In particular, the study of the inner gaseous disc (within a few astronomical units from the central object), namely where accretion and ejection take place, can clarify what mechanisms are at play (e.g. accretion from funnels or through boundary layers, ejection through stellar or MHD discwinds, etc.). This crucial region still remains elusive because of the typically large distance (kiloparsecs; kpc) to HMYSOs and their high visual extinction $\left(A_{V} \geq 50 \mathrm{mag}\right)$. Therefore, near-infrared (NIR) spectro-interferometry is required to achieve milli-arcsecond (mas) spatial resolution and to spectrally resolve the warm gas (few thousand K) that traces such processes. The $\mathrm{CO}$ overtone transitions (or bandheads) in the $K$-band (between 2.29 and $2.5 \mu \mathrm{m}$ ) have been successfully employed to investigate both the kinematics and physics of the inner gaseous disc in HMYSOs (Blum et al. 2004; Bik \& Thi 2004). The modelling of the $\mathrm{CO}$ bandheads profiles at high spectral resolution $(R \geq 10000)$ suggests that such emission comes from warm $(T=2000-5000 \mathrm{~K})$ and dense $\left(n>10^{11} \mathrm{~cm}^{-3}\right)$ gas in Keplerian rotation, within a few astronomical units from the central sources (Ilee et al. 2013), relatively close to the dust sublimation radius. However, this region has not been spatially resolved yet, and therefore its location and position are still uncertain.

Here, we present the first spatially and spectrally resolved observations of the $\mathrm{CO}$ overtone transitions in a HMYSO, namely in NGC 2024 IRS 2, using GRAVITY/VLTI spectrointerferometry. NGC 2024 IRS 2 (hereafter IRS 2) is a wellstudied HMYSO $\left(M_{*} \sim 15 M_{\odot}\right.$; SpT $\sim \mathrm{B} 0 ; M_{\text {disc }} \sim 0.04 M_{\odot}$; Lenorzer et al. 2004; Nisini et al. 1994; Mann et al. 2015). Located in NGC 2024 within the Orion B complex at a distance of $423 \pm 15$ pc (Kounkel et al. 2017), IRS 2 is very bright in the $K$-band $\left(K_{s}=4.585 \mathrm{mag}\right.$; Skrutskie et al. 2006) and its spectrum shows strong CO bandheads (Chandler et al. 1995; Lenorzer et al. 2004), making it an excellent test case for probing the origin of the NIR CO emission in HMYSOs.

\section{Observations and data reduction}

IRS 2 was observed with GRAVITY/VLTI (GRAVITY Collaboration 2017) in the $K$-band $(1.95-2.5 \mu \mathrm{m})$ on 21 January 2019 using the four $8 \mathrm{~m}$ Unit Telescopes (UTs). The target was observed in single-field mode. As both target and nearby stars are not visible in the optical, we used the IR wavefront sensing system CIAO (Coudé Infrared Adaptive Optics; Scheithauer et al. 2016) guiding off-axis on the nearby (4".8) NIR star IRS 2b. Three sets of data (with total integration time of $900 \mathrm{~s}$ each) were acquired. Only UT1-2-3 data are present in the second dataset due to a technical failure at UT4. The complete data $\log$ is reported in Table 1. The data on the fringe tracker (FT) detector were recorded at low spectral resolution $(R \sim 23)$ with a DIT of $0.85 \mathrm{~ms}$ and those of the science (SC) detector at high spectral resolution (HR; $R \sim 4000$, i.e. $\left.\Delta v \sim 70 \mathrm{~km} \mathrm{~s}^{-1}\right)$. The three datasets were reduced using the GRAVITY pipeline (v1.2.1; Lapeyrere et al. 2014). HD 31464 and HD 37491 calibrators were used to retrieve the atmospheric transfer function. The IRS 2 spectrum was obtained by averaging the four HR UT spectra recorded in the three datasets. Standard telluric correction was also applied to the spectrum using HD 31464 (SpT K0 III) as a telluric standard star. The
IRS 2 spectrum was then flux calibrated adopting the 2MASS catalogue value. The spectral wavelength calibration was refined using several telluric absorption lines present along the spectrum. An average shift of $\sim 4.5 \AA$ was applied. To convert the observed wavelengths into radial velocities, we adopted a local standard of rest (lsr) velocity of $6 \mathrm{~km} \mathrm{~s}^{-1}$ (Lenorzer et al. 2004).

\section{Results}

Our GRAVITY/VLTI datasets provide us with the $K$-band spectrum of IRS 2, six (or three for the second dataset taken with 3 UTs) spectrally dispersed visibilities $(V)$ and differential phases (DP), and four (or one for the second dataset) closure phases (CP; see Fig. 1), with rms uncertainties of $\sim 1 \%$ for $V$, $\sim 1^{\circ}$ for $\mathrm{DP}$, and $\sim 2^{\circ}$ for $\mathrm{CP}$.

The IRS 2 spectrum displays a rising continuum with a bright $\operatorname{Br} \gamma(2.166 \mu \mathrm{m})$ line and four overtone CO bandheads (from $v=2-0$ to $v=5-3$, i.e. from 2.29 to $2.39 \mu \mathrm{m}$ ) in emission. No other lines are detected in the spectrum above a threshold of three sigma. The continuum emission is resolved in all the baselines and is slightly asymmetric, displaying small closure phases $\left(\leq 8^{\circ}\right)$ for the triangles with long baselines and closure phases consistent with zero for the shortest baseline triangles $(\leq 60 \mathrm{~m})$. Spectrally dispersed visibilities and DP and CP signatures are detected in both $\mathrm{Br} \gamma$ and $\mathrm{CO}$ lines. Notably, the small continuum asymmetry also affects the observed DP and CP of the lines, causing the redshifted DP peak to be systematically smaller than the blueshifted one and making the $\mathrm{CP}$ value at the line peak smaller than that of the continuum.

In this Letter we report on the interferometric signatures of the $\mathrm{CO}$ bandheads, detected and analysed for the first time in a HMYSO, leaving the $\mathrm{Br} \gamma$ line analysis to a forthcoming publication. Figure 1 shows the interferometric observables (line profiles - inserts A; visibilities - inserts B and C; differential phases - inserts D and E; closure phases - inserts F and G) of the first $\mathrm{CO}$ bandhead $(v=2-0)$ and adjacent continuum for the three runs (panels 1, 2 and 3). The interferometric observables of the other three CO bandheads $(v=3-1, v=4-2$ and $v=5-3)$, which are basically identical to the first one but slightly more noisy, are shown in Figs. A.1-A.3. Visibility values, $V$, around each bandhead peak are larger than the continuum visibilities at all the six baselines in the three runs (see inserts $\mathrm{B}$ and $\mathrm{C}$ in Fig. 1 and Figs A.1-A.3), indicating that the CO-emitting region, though spatially resolved, is more compact than the continuum. $\mathrm{DP}$ at four of the six baselines (or at all the three baselines of the second dataset) display an asymmetric "S" shape with values up to $12^{\circ}$ and small $\left(\geq 2^{\circ}\right)$ values at the short baselines as well as at the intermediate baselines with PA around $80^{\circ}$, indicating a clockwise rotating disc with a major axis PA close to $170^{\circ}$.

\subsection{Continuum-emitting region}

To estimate the size, inclination $(i)$, and position angle of the dusty disc, we fit the continuum visibilities recorded with the GRAVITY FT using a simple geometric model, which assumes a point-like star and a resolved circumstellar disc, as in Lazareff et al. (2017). No additional extended halo component is required to fit the data, so we do not include it in the model. This is likely because the IRS 2 outflow cavity walls (the main source of such an extended halo in HMYSOs) are located well beyond the UT field of view (FoV); $>200$ mas vs. $~ 40$ mas. Therefore, the complex visibility $(V)$ at spatial frequency $(u, v)$ and at a 
GRAVITY Collaboration: Spatially resolved CO bandhead emission in NGC 2024 IRS 2

Table 1. Observation log of the VLTI GRAVITY+UT high-resolution $(R \sim 4000)$ observations of NGC 2024 IRS 2.

\begin{tabular}{lccccccc}
\hline \hline $\begin{array}{l}\text { Date UT } \\
\text { yyyy-mm-dd hh:mm }\end{array}$ & $\begin{array}{c}\text { Tot. Int. } \\
{[\mathrm{s}]}\end{array}$ & $\begin{array}{c}\text { DIT }^{(a)} \\
{[\mathrm{s}]}\end{array}$ & $\begin{array}{c}\text { NDIT } \\
{[\mathrm{s}]}\end{array}$ & $\begin{array}{c}\text { Proj. baselines } \\
{[\mathrm{m}]}\end{array}$ & $\begin{array}{c}\text { PA }^{(c)} \\
{[\mathrm{o}]}\end{array}$ & Calibrator & $\begin{array}{c}\text { UD diameter }^{(d)} \\
{[\mathrm{mas}]}\end{array}$ \\
\hline 2019-01-21 05:04 & 900 & 30 & 10 & $45,45,56,72,101,119$ & $298,46,36,82,40,62$ & HD 31464, HD 37491 & $0.186 \pm 0.004,0.464 \pm 0.018$ \\
2019-01-21 05:27 & 900 & 30 & 10 & $44,56,100$ & $45,36,40$ & HD 31464, HD 37491 & $0.186 \pm 0.004,0.464 \pm 0.018$ \\
2019-01-21 06:00 & 900 & 30 & 10 & $34,43,55,56,98,103$ & $309,43,35,80,39,53$ & HD 31464, HD 37491 & $0.186 \pm 0.004,0.464 \pm 0.018$ \\
\hline
\end{tabular}

Notes. ${ }^{(a)}$ Detector integration time per interferogram. ${ }^{(b)}$ Number of interferograms. ${ }^{(c)}$ Baseline position angle (PA, from N to E) from the shortest to longest baseline. ${ }^{(d)}$ The calibrator uniform-disc (UD) diameter ( $K$ band) was taken from Chelli et al. (2016).

certain wavelength $(\lambda)$ consists of two components: $V(u, v, \lambda)=$ $f_{\mathrm{s}}(\lambda)+f_{\mathrm{d}}(\lambda) V_{\mathrm{d}}(u, v, \lambda)$, where $f_{\mathrm{s}}$ and $f_{\mathrm{d}}$ are the stellar and disc contributions to the continuum flux $\left(f_{\mathrm{s}}+f_{\mathrm{d}}=1\right), V_{\mathrm{d}}$ is the disc visibility, and the stellar visibility is assumed to be 1 , as the star is not resolved at our spatial resolution $(\sim 0.17$ mas is the expected diameter of a B0 zero age main sequence - ZAMS star at $423 \mathrm{pc}$ ). A stellar contribution factor to the continuum flux of $f_{\mathrm{s}}=0.07 \pm 0.04$ was first estimated, assuming a stellar spectral type of B0 and $A_{V}$ of $24 \mathrm{mag}$ (Lenorzer et al. 2004). The $f_{\mathrm{s}}$ value, together with its uncertainty, is used as a starting value in the interferometric fit, but is kept as a free parameter during the visibility fitting process.

We use the fitting tool described in Lazareff et al. (2017) to test different models in their ability to fit both $V^{2}$ and CP. We test ellipsoids and rings with Gaussian and non-Gaussian radial brightness distributions. The free parameters for the ellipsoid models are the flux contributions of $f_{\mathrm{s}}$ and $f_{\mathrm{d}}$, the flattening as cos $i$, PA, the weighting for the radial brightness distribution Lor, which varies from a purely Gaussian to a purely Lorentzian distribution, and the half-flux semi-major axis $a$. The non-Gaussian models lead to $\chi_{\mathrm{r}}^{2}$ values closer to 1 . The ellipsoid and ring models converge towards the same set of parameters. Our best fit ellipsoid model $\left(\chi_{\mathrm{r}}^{2}=0.33\right)$ provides an inclination of $34^{\circ} \pm 1^{\circ}$, a PA of about $166^{\circ} \pm 1^{\circ}$, and a disc diameter of $3.99 \pm 0.09$ mas $(1.69 \pm 0.04 \mathrm{au}$ ) as reported in Table 2 (see Fig. B.1 for the continuum fit and Table B.1 for the whole set of modelled parameters). Notably, the derived $i$ value is equal to that inferred by Chandler et al. (1995) $\left(33^{\circ}\right)$, who fit the $v=2-0$ $\mathrm{CO}$ bandhead (observed at high-spectral resolution $\mathcal{R} \sim 15000$ ) with a disc in Keplerian rotation. The disc contribution dominates the continuum emission in the $K$-band with a flux contribution of about $91 \%$, in full agreement with the estimate derived from the spectral type. In addition, we are able to correctly model the small closure phase signals with a skewed rim, originating from a pure inclination effect, with its maximum brightness roughly located westwards.

\subsection{CO continuum-subtracted visibilities and closure phases}

Both size and geometry of the CO-emitting region can be determined from the pure (or continuum compensated) $\mathrm{CO}$ bandhead visibilities $\left(V_{\mathrm{CO}}\right)$. These are estimated by subtracting the continuum contribution to the total line visibilities and by taking into account the line photocentre shifts (Weigelt et al. 2007):

$V_{\mathrm{CO}}=\frac{\sqrt{\left|F_{\text {tot }} V_{\text {tot }}\right|^{2}+\left|F_{\text {cont }} V_{\text {cont }}\right|^{2}-2 F_{\text {tot }} V_{\text {tot }} F_{\text {cont }} V_{\text {cont }} \cos \phi}}{F_{\text {line }}}$,

where $F_{\text {tot }}=F_{\text {cont }}+F_{\text {line }}, F_{\text {line }}$, and $F_{\text {cont }}$ are the total, line, and continuum fluxes, respectively; $V_{\text {cont }}$ and $V_{\text {tot }}$ are the measured continuum and total visibilities, respectively; and $\phi$ is the differential phase. We compute $V_{\mathrm{CO}}$ for the four bandheads, in the three spectral channels around the bandhead peak, namely those with line-to-continuum ratio larger than 30\%. Errors are estimated taking into account the uncertainties on the continuum and line fluxes, on the total visibilities, and on the differential phases for each spectral channel. Within the error bars, the three values are the same, and therefore we average the results obtaining less noisy values with average errors $\left(\Delta V_{\mathrm{CO}}\right)$ of 0.03 . $V_{\mathrm{CO}}$ and $\Delta V_{\mathrm{CO}}$ per baseline and per bandhead are reported in Table C.1, along with the $(u, v)$ values, projected baseline length, $\mathrm{PA}$, and the UT telescope configuration. To determine the size, inclination, and position angle of the $\mathrm{CO}$-emitting region, we then fit the $V_{\mathrm{CO}}$ datapoints in the $(u, v)$ plane using a bidimensional Gaussian with $\mathrm{FWHM}_{\mathrm{CO}}, i_{\mathrm{CO}}$ and $\mathrm{PA}_{\mathrm{CO}}$ as free parameters. To derive the best values we use our own Python program based on the Monte-Carlo and Markov chain (MCMC) code emcee (Foreman-Mackey et al. 2013, see also details on the method in Appendix C). We first model the visibilities of each single bandhead, the fit of which provides, within the error bars, very similar results in size (see Table 2 ). As the different bandheads are excited at different temperatures, the latter suggests that the radial extent $(\Delta R)$ of the CO-emitting region must be relatively small $(\Delta R / R \leq 20 \%)$.

To improve our results, we fit the whole set of visibilities, assuming that the bandhead-emitting regions have the same size and geometry. The best model $\left(\chi_{\mathrm{r}}^{2}=1.34\right.$; see MCMC marginal posterior distributions in Fig. C.1) is shown in Fig. 2, which reports the bidimensional Gaussian projected on the $(u, v)$ plane along with the observed visibilities and their uncertainties. The CO-emitting region has a diameter of $2.74 \pm_{0.07}^{0.08}$ mas $(1.16 \pm 0.03 \mathrm{au})$, namely it is located in the inner gaseous disc within the dusty rim, with $i_{\mathrm{CO}}\left(32^{\circ} \pm 3^{\circ}\right)$ and $\mathrm{PA}_{\mathrm{CO}}\left(168^{\circ} \pm_{4^{\circ}}{ }^{\circ}\right)$, matching the geometry of the dusty disc (see Table 2) and indicating that both discs are coplanar. Notably, the inferred $\mathrm{CO}$ radius value $(0.58 \mathrm{au})$ is within the range of values $(0.28-$ $0.84 \mathrm{au}$ ) estimated by Chandler et al. (1995).

To retrieve any asymmetry from the $\mathrm{CO}$-emitting region, we remove the continuum contribution from the line closure phase of each UT triangle, obtaining the so-called closure differential phase (CDP), which is the closure of the pure differential phases of the CO bandheads. We compute the CDP for the seven triangles available and for the first four bandheads around their peaks (averaging three to five spectral channels), namely where the line-to-continuum ratio is larger than 1.3. As a result, the $\mathrm{CDP}$ of the $\mathrm{CO}$-emitting region is $\sim 0^{\circ}$ (within the uncertainties, which range form $\sim 5^{\circ}$ to $\sim 10^{\circ}$ for the first and the fourth bandhead, respectively). This indicates that the $\mathrm{CO}$-emitting region is symmetric around the central source, and its small CP signatures (see panels G in Fig. 1 and Figs. A.1-A.3) arise from the continuum asymmetry. 


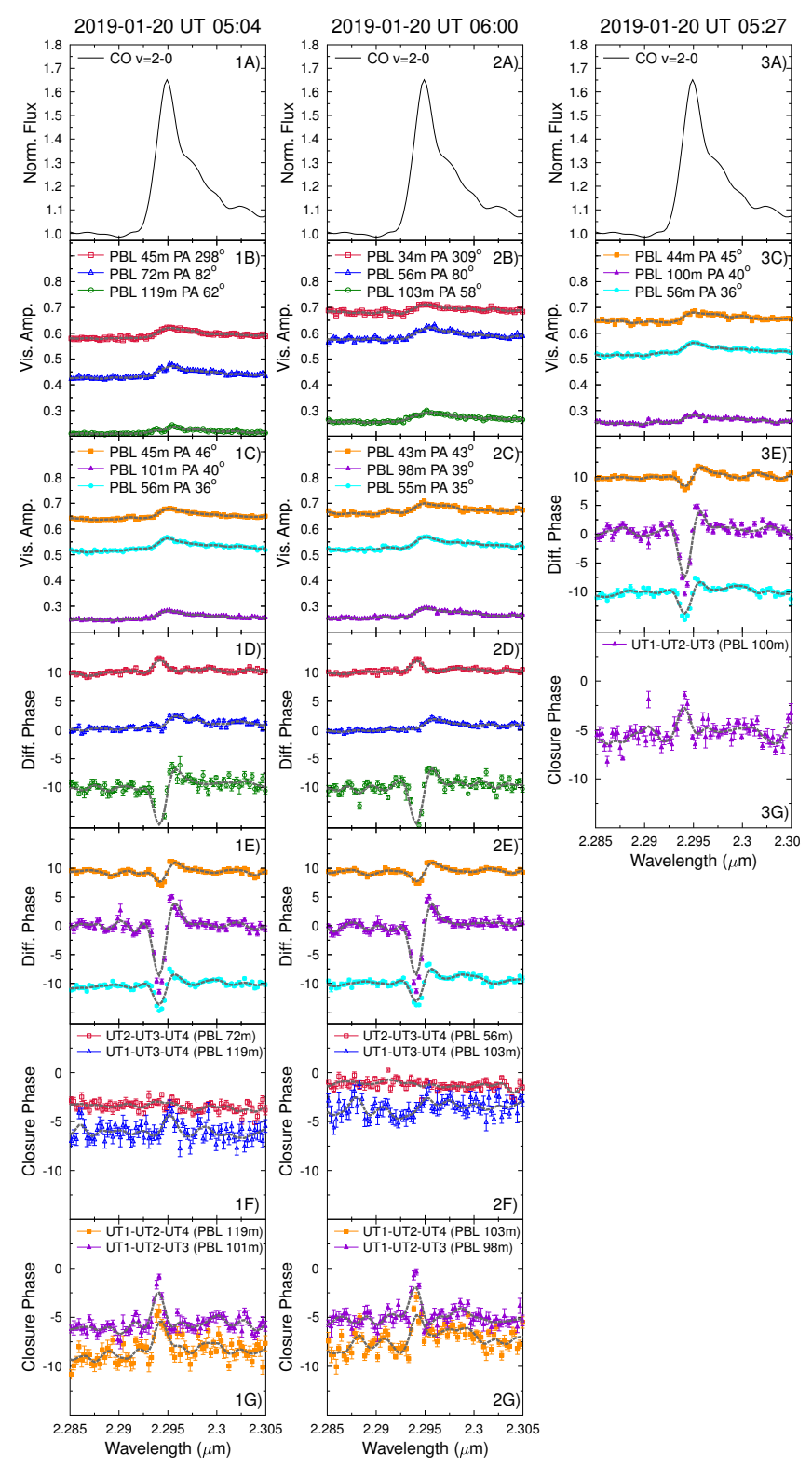

Fig. 1. Left, panel 1: interferometric measurements of the CO $v=2-0$ bandhead in NGC 2024 IRS2 for run 1 (inserts 1A-1G). From top to bottom: total flux normalised to continuum (1A); wavelength-dependent visibilities for UT 3-4, 2-4, 1-4 (1B) and for UT 2-3, 1-3, 1-2 (1C); differential phases for UT $3-4,2-4,1-4$ (1D) and for UT $2-3,1-3$, 1-2 (1E); and closure phases for UT 2-3-4, 1-3-4 triplets (1F) 1-2-4 and 1-2-3 triplets (1G). Middle, panel 2: interferometric measurements of the CO $v=2-0$ and $v=3-1$ bandheads in NGC 2024 IRS2 for run 3 (inserts 2A-2G). Right, panel 3: interferometric measurements of the CO $v=2-0$ and $v=3-1$ bandheads in NGC 2024 IRS2 for run 2 (inserts 3A-3G). From top to bottom: total flux normalised to continuum (3A); wavelength-dependent visibilities for UT 2-3, 1-3, 1-2 (3C); differential phases for UT 2-3, 1-3, 1-2 (3E); and closure phase for UT $1-2-3(3 G)$. For clarity, the differential phases of the first and last baselines are shifted by $+10^{\circ}$ and $-10^{\circ}$, respectively.

\subsection{CO physical parameters}

We use a CO local thermodynamic equilibrium (LTE) model (see Koutoulaki et al. 2019, for a detailed description of model, code and error estimates) to derive the main physical parameters of the gas. We model the $\mathrm{CO}$ bandhead profiles with a single ring in LTE with four free parameters: temperature $T_{\mathrm{CO}}$, column
Table 2. Diameter, inclination, and position angle derived from the best fit of continuum and $\mathrm{CO}$ bandheads.

\begin{tabular}{lcccc}
\hline \hline Continuum & $\begin{array}{c}\text { diameter } \\
{[\mathrm{mas}]}\end{array}$ & $\begin{array}{c}\text { diameter } \\
{[\mathrm{au}]}\end{array}$ & $\begin{array}{c}i \\
{\left[{ }^{\circ}\right]}\end{array}$ & $\begin{array}{c}\text { PA } \\
{\left[{ }^{\circ}\right]}\end{array}$ \\
\hline & $3.99 \pm_{0.1}^{0.08}$ & $1.69 \pm_{0.04}^{0.03}$ & $34 \pm 1$ & $166 \pm 1$ \\
\hline Bandhead & & & & \\
\hline All & $2.74 \pm_{0.07}^{0.08}$ & $1.16 \pm 0.03$ & $32 \pm 3$ & $168 \pm_{4}^{5}$ \\
$v=2-0$ & $2.9 \pm_{0.2}^{0.1}$ & $1.21 \pm_{0.08}^{0.04}$ & $33 \pm_{8}^{5}$ & $159 \pm_{5}^{8}$ \\
$v=3-1$ & $2.6 \pm 0.1$ & $1.10 \pm 0.04$ & $28 \pm_{7}^{6}$ & $177 \pm_{12}^{14}$ \\
$v=4-2$ & $2.8 \pm 0.1$ & $1.18 \pm 0.04$ & $32 \pm_{5}^{4}$ & $169 \pm_{7}^{11}$ \\
$v=5-3$ & $2.5 \pm 0.1$ & $1.06 \pm 0.04$ & $33 \pm_{6}^{4}$ & $187 \pm_{12}^{9}$ \\
\hline
\end{tabular}

Notes. $1 \sigma$ uncertainties are reported.

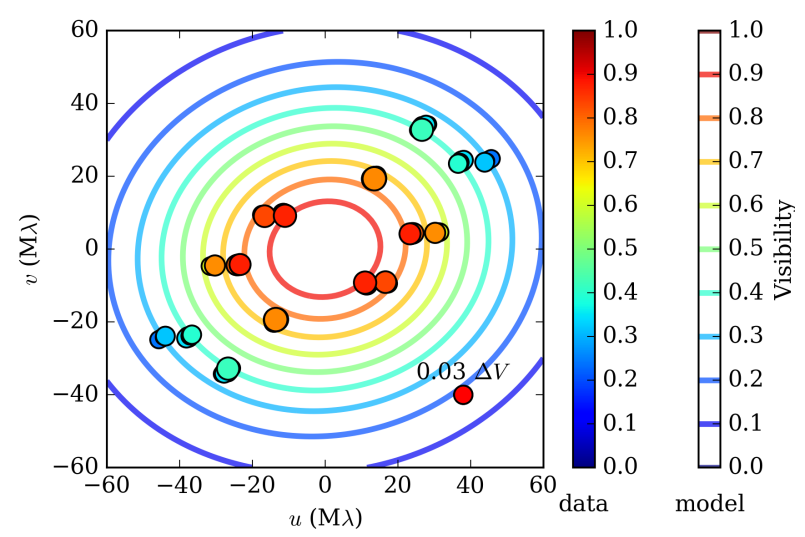

Fig. 2. CO $2 \mathrm{D}$ Gaussian model (coloured ellipses) in the $(u, v)$ plane for the observed visibilities of the four bandheads (coloured circles). Visibility values (from 0 to 1 ) of both model and observations are shown in scale from blue to red. Visibility uncertainties are represented with different diameters (see red circle at the bottom right of the plot for an uncertainty average value of 0.03 ).

density $N_{\mathrm{CO}}$, turbulence velocity of the gas $\Delta v$, and projected Keplerian velocity $\left(v_{K} \sin i\right.$, where $i$ is the inclination of the disc plane with respect to the sky plane). A large grid of models was computed ranging over the free parameter space and then each resulting spectrum was convolved to the GRAVITY spectral resolution. Our model is able to reproduce both the peaks and tails of the four bandheads very well, although some portions of the tails are clearly missed due to the presence of strong telluric features. Figure 3 shows the spectrum of the first four CO bandheads (black curve) overplotted over our best model (red curve) with the following parameters: $T_{\mathrm{CO}}=2800_{-200}^{+300} \mathrm{~K}, N_{\mathrm{CO}}=$ $\left(5_{-1}^{+5}\right) \times 10^{20} \mathrm{~cm}^{-2}, \Delta v=1_{-0.5}^{+1} \mathrm{~km} \mathrm{~s}^{-1}$, and $v_{K} \sin i=84_{-20}^{+10} \mathrm{~km} \mathrm{~s}^{-1}$. By measuring inclination and $\mathrm{CO}$ position from our interferometric data and the $v_{K} \sin i$ from the spectral fit, the stellar mass can be derived. It is worth noting that such a measurement is not possible otherwise as the photospheric veiling in HMYSO is too high for a proper spectral-type estimate and is more accurate than those derived with ALMA, for example, which include the whole disc mass. If we adopt the $i$ value from the dusty disc, we obtain an estimate of the Keplerian velocity of $v_{K} \sim 150 \mathrm{~km} \mathrm{~s}^{-1}$, which at $0.58 \mathrm{au}$ implies a central mass of $M_{*} \sim 14.7_{-3.6}^{+2} M_{\odot}$. This corresponds to a SpT $=\mathrm{B} 0.5 \pm 0.5$ (i.e. $T_{\text {eff }}=29000_{-3000}^{+2500} \mathrm{~K}$; Pecaut \& Mamajek 2013), assuming that IRS 2 is on the ZAMS. 


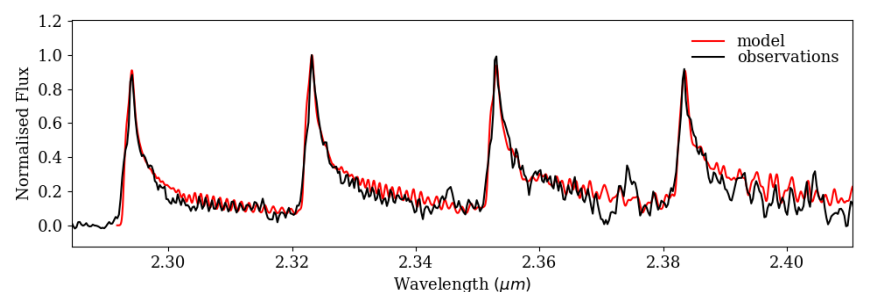

Fig. 3. GRAVITY spectrum of the first four CO bandheads (in black) overplotted on our LTE model (in red). The GRAVITY spectrum is continuum subtracted and normalised to the peak of the second bandhead.

\section{Origin of the CO bandheads in NGC 2024 IRS 2}

Our interferometric results demonstrate that the $\mathrm{CO}$ bandheads are emitted in the inner gaseous disc (located at 0.58 au from the star), more specifically in a dust-free region well within the dust sublimation radius (located at 0.85 au from IRS 2). We infer that IRS 2 is a $\sim 15 M_{\odot}$ star with $T_{\text {eff }} \sim 29000 \mathrm{~K}$ on ZAMS. As the inner gaseous region is free from dust grains, the $\mathrm{CO}$ molecules should be photodissociated by the stellar UV photons. It is worth asking why the photo-dissociation does not happen and whether the CO-emitting region is in the disc midplane or more close to its surface. To answer the first question, we note that the IRS 2 mass accretion rate $\left(\dot{M}_{\text {acc }}\right)$ is $\sim 5 \times 10^{-7} M_{\odot} \mathrm{yr}^{-1}$ (Chandler et al. 1995; Lenorzer et al. 2004), which is high enough to make the gaseous disc optically thick (see Dullemond $\&$ Monnier 2010, and references therein). Therefore, the very inner gaseous disc should be able to shield the $\mathrm{CO}$-emitting gas. Moreover, the observed CO column density $\left(\sim 5 \times 10^{20} \mathrm{~cm}^{-2}\right)$ is much higher than the value needed for the $\mathrm{CO}$ molecules to selfshield $\left(\sim 10^{15} \mathrm{~cm}^{-2}\right.$; see van Dishoeck \& Black 1988; Bik \& Thi 2004). To answer the second question, we consider an optically thick Shakura-Sunyaev-type accretion disc around a B0.5 star on the ZAMS (with parameters $M_{*}=14.7 M_{\odot}, R_{*}=7 R_{\odot}$, $T_{\text {eff }}=29000 \mathrm{~K}, \dot{M}_{\text {acc }}=5 \times 10^{-7} M_{\odot} \mathrm{yr}^{-1}$ ). At 0.58 au (i.e. where the $\mathrm{CO}$ is located), we infer surface density $(\Sigma)$ values ranging from $10^{3}$ to $10^{4} \mathrm{~g} \mathrm{~cm}^{-2}$ (using Eq. (16) of Dullemond \& Monnier 2010, and varying the turbulent viscosity coefficient $\alpha$ from 0.1 to 0.01 ). A midplane temperature of $\sim 2000 \mathrm{~K}$ can also be estimated (using Eq. (15) of Dullemond \& Monnier 2010), assuming that the disc is heated by stellar radiation rather than viscous accretion, which becomes relevant at much higher mass accretion rates for HMYSOs (see e.g. Fedriani et al. 2020). In comparison, the total column density traced by the $\mathrm{CO}$ (assuming a factor of $10^{4}$ between the $\mathrm{CO}$ and the total gas column density) is $8_{-2}^{+8} \mathrm{~g} \mathrm{~cm}^{-2}$, which is three or four orders of magnitude lower than what is predicted for the disc midplane. In addition, the observed CO temperature $\left(T_{\mathrm{CO}}=2800_{-200}^{+300} \mathrm{~K}\right)$ is higher than that predicted for the disc midplane but lower than what is expected on the disc surface $(\sim 4500 \mathrm{~K})$. At this temperature, the molecule is completely destroyed (Bosman et al. 2019). This reasoning indicates that the $\mathrm{CO}$-emitting region is located well above the midplane but below the disc surface.

\section{Conclusions}

The main results of the first spectro-interferometric observations of the CO bandheads in the HMYSO NGC 2024 IRS 2 are the following.
1. The $\mathrm{CO}$ overtone (located at $0.58 \pm 0.02$ au from the star) is emitted from a dust-free region in the inner gaseous disc, which is coplanar with the inner dusty disc (located at $0.85 \pm 0.02 \mathrm{au}$, with $i=34^{\circ} \pm 1^{\circ}$ and $\left.\mathrm{PA}=166^{\circ} \pm 1^{\circ}\right)$ and of relatively small radial extent $(\Delta R / R \leq 20 \%)$. The emitting region is located well above the disc midplane but below the disc surface.

2. By modelling the $\mathrm{CO}$ spectrum, the following physical parameters are inferred: $T_{\mathrm{CO}}=2800_{-200}^{+300} \mathrm{~K}, N_{\mathrm{CO}}=\left(5_{-1}^{+5}\right) \times$ $10^{20} \mathrm{~cm}^{-2}, \Delta v=1_{-0.5}^{+1} \mathrm{~km} \mathrm{~s}^{-1}$, and $v_{K} \sin i=84_{-20}^{+10} \mathrm{~km} \mathrm{~s}^{-1}$.

3. By combining inclination and $\mathrm{CO}$ position from our interferometric data and the $v_{K} \sin i$ from the spectral fit, we provide a direct measurement of the stellar mass $\left(M_{*} \sim 14.7_{-3.6}^{+2} M_{\odot}\right)$ in a HMYSO.

Acknowledgements. This paper is based on observations made with ESO Telescopes at the La Silla Paranal Observatory under programme IDs 0102.C0408(C). We thank the technical, administrative, and scientific staff of the participating institutes and the observatory for their extraordinary support during the development, installation, and commissioning of GRAVITY. A.C.G. wishes to thank Antonella Natta for her useful insights and comments. A.C.G. and T.P.R. have received funding from the European Research Council (ERC) under the European Union's Horizon 2020 research and innovation programme (grant agreement No. 743029). R.F. acknowledges support from Science Foundation Ireland (grant 13/ERC/12907) and from a Chalmers Initiative on Cosmic Origins (CICO) postdoctoral fellowship. M.K. is funded by the Irish Research Council (IRC), grant GOIPG/2016/769 and Science Foundation Ireland (grant 13/ERC/12907). R.G.L has received funding from Science Foundation Ireland under Grant No. 18/SIRG/5597. K.P. acknowledges the funding of the French National Program of Stellar Physics (PNPS) and the grant from LabEx OSUG@2020 (Investissements d'avenir - ANR10LABX56), A.A., P.G., P.G. were supported by Fundacão para a Ciência e a Tecnologia, with grants reference UID/FIS/00099/2013 and SFRH/BSAB/142940/2018. This research has made use of the Jean-Marie Mariotti Center Aspro and SearchCal services (http://www . jmmc.fr/), and of CDS Astronomical Databases SIMBAD and VIZIER (http: //cdsweb.u-strasbg.fr/).

\section{References}

Beltrán, M. T., \& de Wit, W. J. 2016, A\&ARv, 24, 6 Bik, A., \& Thi, W. F. 2004, A\&A, 427, L13

Blum, R. D., Barbosa, C. L., Damineli, A., Conti, P. S., \& Ridgway, S. 2004, ApJ, 617, 1167

Bosman, A. D., Banzatti, A., Bruderer, S., et al. 2019, A\&A, 631, A133

Chandler, C. J., Carlstrom, J. E., \& Scoville, N. Z. 1995, ApJ, 446, 793

Chelli, A., Duvert, G., Bourgès, L., et al. 2016, A\&A, 589, A112

Dullemond, C. P., \& Monnier, J. D. 2010, ARA\&A, 48, 205

Fedriani, R., Caratti o Garatti, A., Koutoulaki, M., et al. 2020, A\&A, 633, A128

Foreman-Mackey, D., Hogg, D. W., Lang, D., \& Goodman, J. 2013, PASP, 125, 306

GRAVITY Collaboration (Abuter, R., et al.) 2017, A\&A, 602, A94

Ilee, J. D., Wheelwright, H. E., Oudmaijer, R. D., et al. 2013, MNRAS, 429, 2960

Kounkel, M., Hartmann, L., Loinard, L., et al. 2017, ApJ, 834, 142

Koutoulaki, M., Facchini, S., Manara, C. F., et al. 2019, A\&A, 625, A49

Lapeyrere, V., Kervella, P., Lacour, S., et al. 2014, in Optical and Infrared Interferometry IV, Proc. SPIE, 9146, 91462D

Lazareff, B., Berger, J. P., Kluska, J., et al. 2017, A\&A, 599, A85

Lenorzer, A., Bik, A., de Koter, A., et al. 2004, A\&A, 414, 245

Mann, R. K., Andrews, S. M., Eisner, J. A., et al. 2015, ApJ, 802, 77

Nisini, B., Smith, H. A., Fischer, J., \& Geballe, T. R. 1994, A\&A, 290, 463

Pecaut, M. J., \& Mamajek, E. E. 2013, ApJS, 208, 9

Scheithauer, S., Brandner, W., Deen, C., et al. 2016, in Adaptive Optics Systems

V, SPIE Conf. Ser., 9909, 99092L

Skrutskie, M. F., Cutri, R. M., Stiening, R., et al. 2006, AJ, 131, 1163

van Dishoeck, E. F., \& Black, J. H. 1988, ApJ, 334, 771

Weigelt, G., Kraus, S., Driebe, T., et al. 2007, A\&A, 464, 87 


\section{Appendix A: CO interferometric observables}

Interferometric measurements of the $\mathrm{CO} v=3-1, v=4-2$ and $v=5-3$ bandheads are reported in Figs. A.1, A.2, and A.3, respectively.
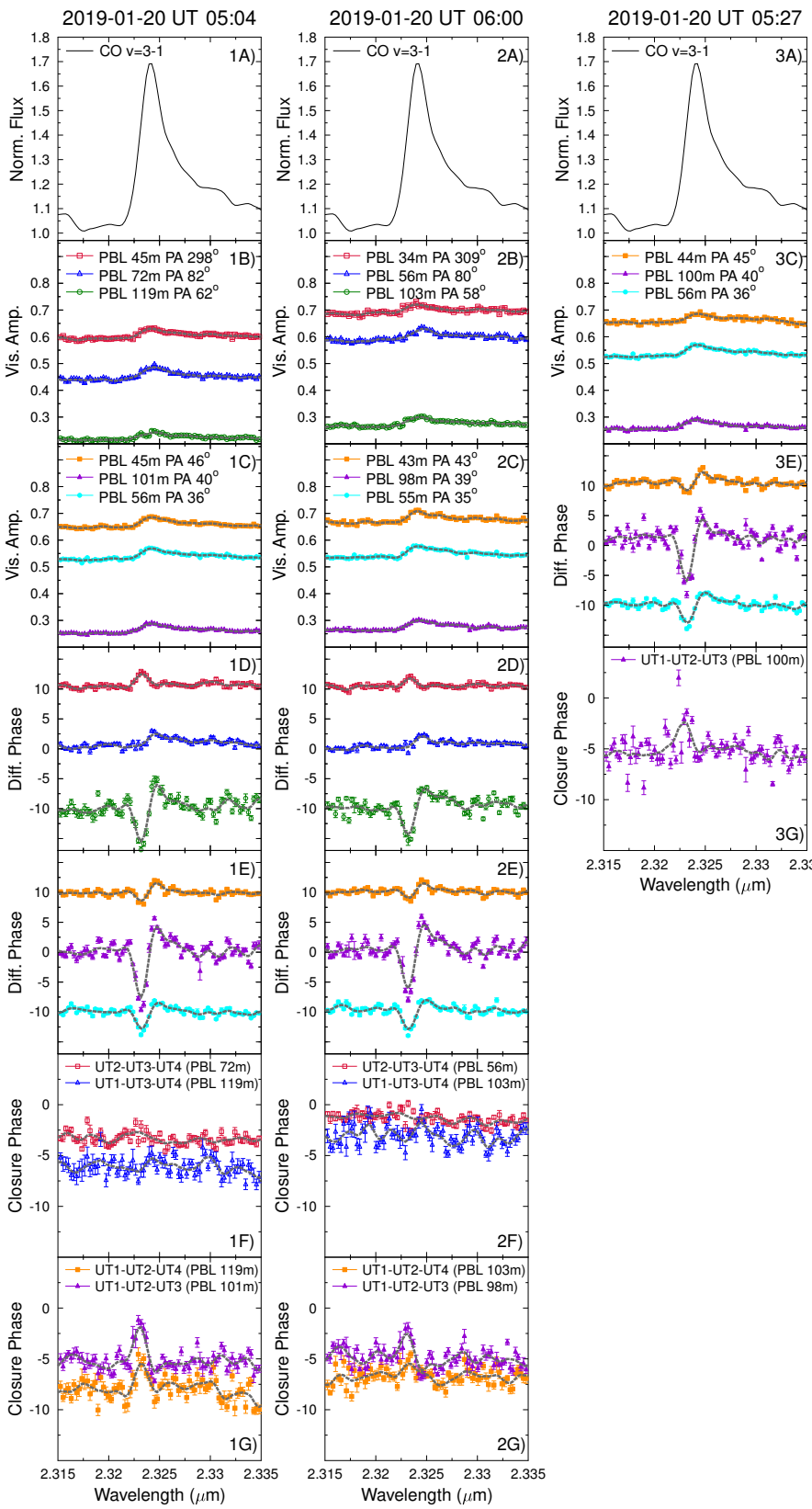

Fig. A.1. Left, panel 1: interferometric measurements of the $\mathrm{CO} v=3-1$ bandhead in NGC 2024 IRS2 for run 1 (inserts 1A-1G). From top to bottom: total flux normalised to continuum (1A); wavelength-dependent visibilities for UT 3-4, 2-4, 1-4 (1B) and for UT 2-3, 1-3, 1-2 (1C); differential phases for UT 3-4, 2-4, 1-4 (1D) and for UT 2-3, 1-3, 1-2 (1E); closure phases for UT 2-3-4, 1-3-4 triplets (1F) 1-2-4 and 1-2-3 triplets $(1 \mathrm{G})$. Middle, panel 2: interferometric measurements of the CO $v=4-2$ and $v=5-3$ bandheads in NGC 2024 IRS2 for run 3 (inserts 2A-2G). Right, panel 3: interferometric measurements of the CO $v=4-2$ and $v=5-3$ bandheads in NGC 2024 IRS2 for run 2 (inserts 3A-3G). From top to bottom: total flux normalised to continuum (3A); wavelength-dependent visibilities for UT 2-3, 1-3, 1-2 (3C); differential phases for UT 2-3, 1-3, 1-2 (3E); and closure phase for UT $1-2-3$ (3G). For clarity, the differential phases of the first and last baselines are shifted by $+10^{\circ}$ and $-10^{\circ}$, respectively.
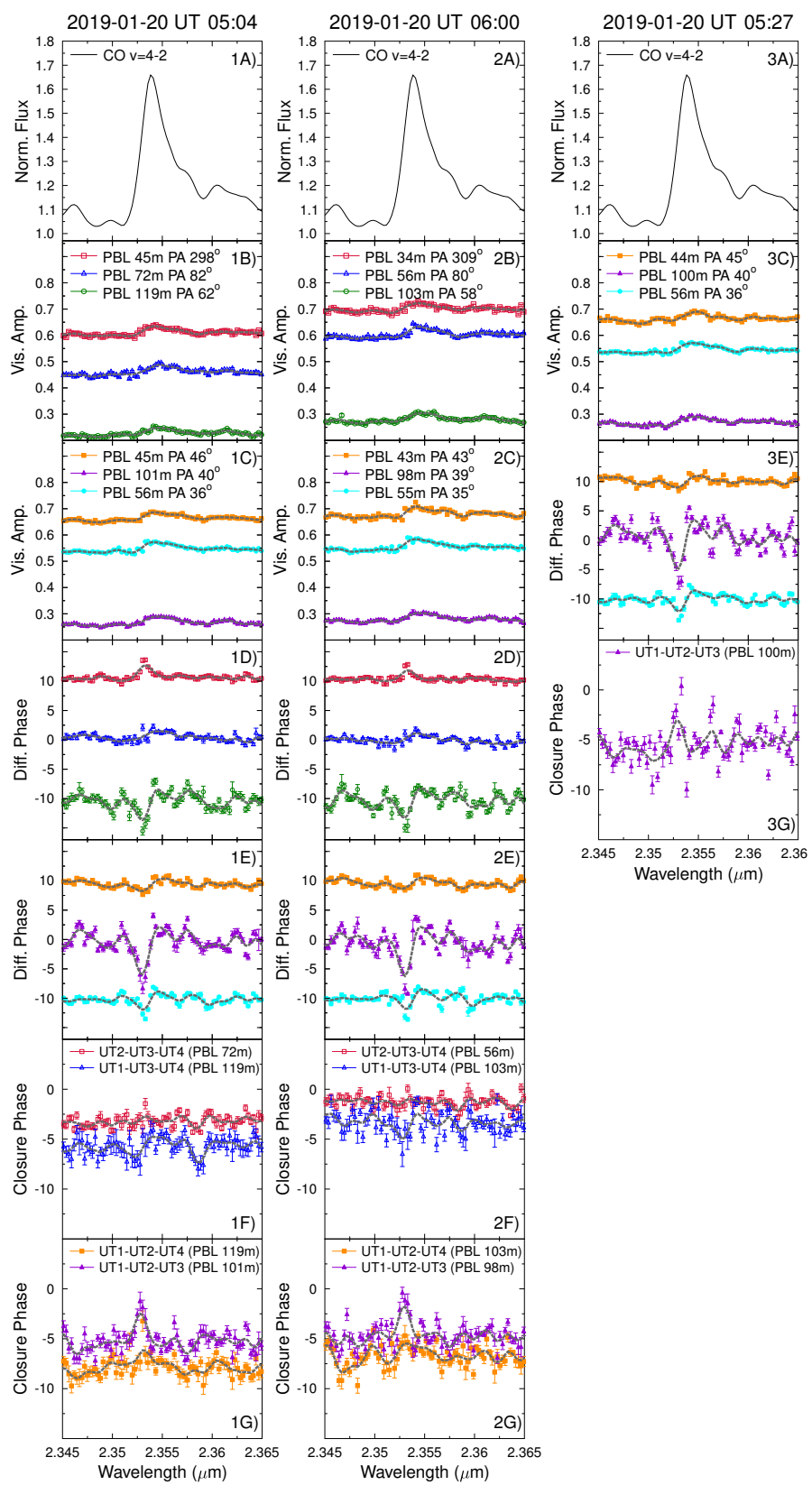

Fig. A.2. Same as Fig. A.1 but for the $\mathrm{CO} v=4-2$ bandhead. 

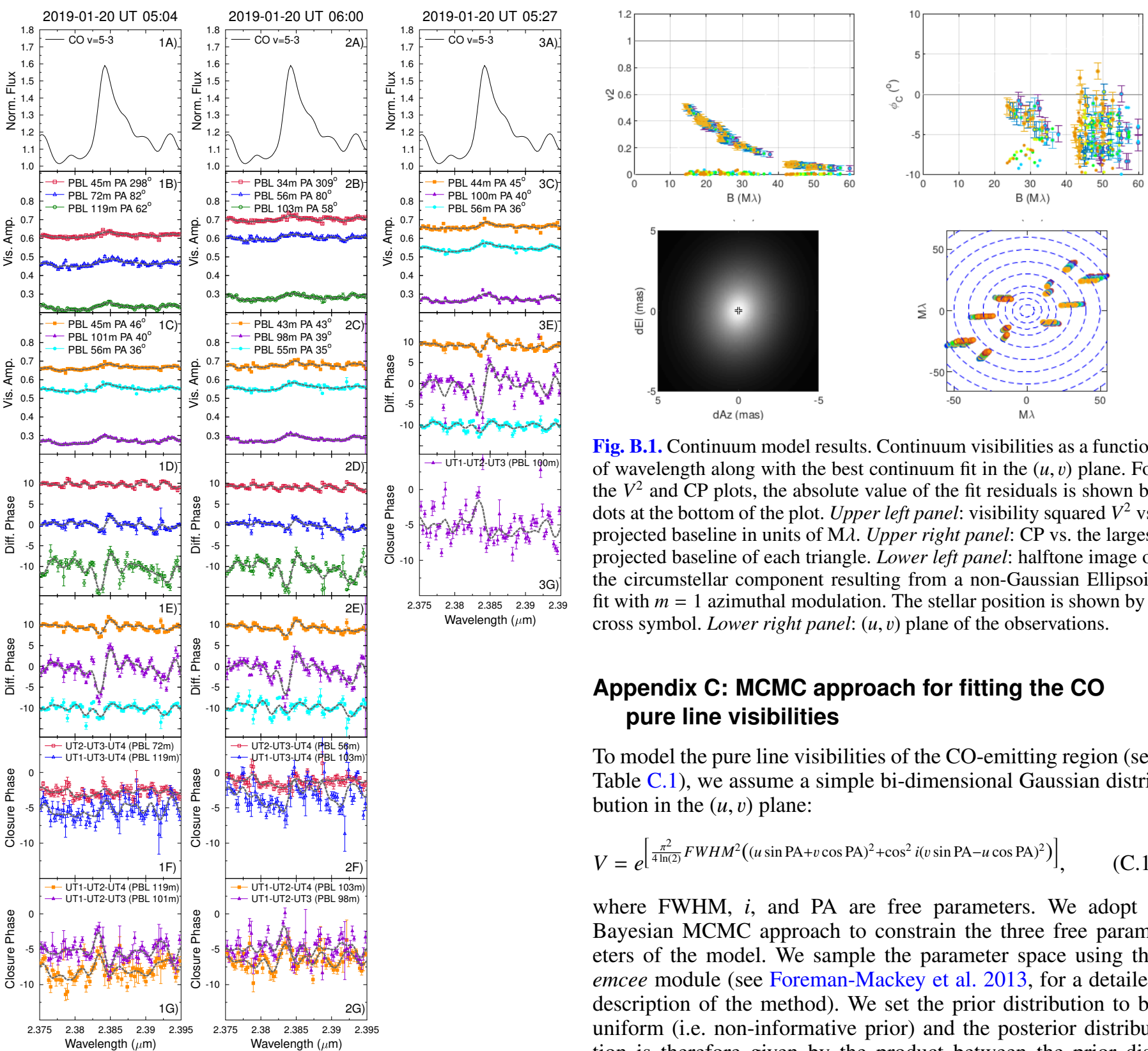

$3 G)$

Wavelength $(\mu \mathrm{m})$ fit fit with $m=1$ azimuthal modulation. The stellar position is shown by a

\section{Appendix C: MCMC approach for fitting the CO pure line visibilities}

To model the pure line visibilities of the CO-emitting region (see Table C.1), we assume a simple bi-dimensional Gaussian distribution in the $(u, v)$ plane:

$V=e^{\left[\frac{\pi^{2}}{4 \ln (2)} F W H M^{2}\left((u \sin \mathrm{PA}+v \cos \mathrm{PA})^{2}+\cos ^{2} i(v \sin \mathrm{PA}-u \cos \mathrm{PA})^{2}\right)\right]}$,

where FWHM, $i$, and PA are free parameters. We adopt a Bayesian MCMC approach to constrain the three free parameters of the model. We sample the parameter space using the emcee module (see Foreman-Mackey et al. 2013, for a detailed description of the method). We set the prior distribution to be uniform (i.e. non-informative prior) and the posterior distribution is therefore given by the product between the prior distribution function and the likelihood function given by $\chi^{2}=$ $\sum\left(V_{\text {obs }}-V_{\text {mod }}\right)^{2} / \sigma_{V}^{2}$ being $\sigma_{V}$ the variance of the data. We ran the MCMC with 1000 walkers and for 1000 steps. We set a burn-in period of $10 \%$, to account for the warm-up period of the chain. To avoid local minima, we first explore a large range of the space parameters (i.e. $F W H M \in[1,4$ mas $], i \in\left[0^{\circ}, 90^{\circ}\right]$, $\mathrm{PA} \in\left[0^{\circ}, 180^{\circ}\right]$; see marginal posterior distributions in Fig. C.1, left panel) and then we reduce the range around the values obtained from those distributions (i.e. $F W H M \in$ [2.5, 3.1 mas], $i \in\left[25^{\circ}, 45^{\circ}\right]$, PA $\in\left[150^{\circ}, 180^{\circ}\right]$; see posterior distributions in Fig. C.1, right panel). Assuming a Gaussian distribution, the most likely value is that of the 50th percentile, whereas the $1 \sigma$ uncertainty is given by the values falling in the 16th and 84th percentiles, represented in the histograms with vertical dashed lines. These values are given on top of each marginal posterior distribution plot. We then calculate the $\chi_{\mathrm{r}}^{2}$ for the most likely values.

Notes. ${ }^{(a)}$ Weighting for radial distribution and ranges between 0 (for a Gaussian radial distribution) and 1 (for a Lorentzian radial distribution). 
A\&A 635, L12 (2020)

Table C.1. CO bandheads pure line visibilities.

\begin{tabular}{|c|c|c|c|c|c|c|c|}
\hline $\begin{array}{l}u \\
(M \lambda)\end{array}$ & $\begin{array}{c}v \\
(M \lambda)\end{array}$ & $V_{\mathrm{CO}}$ & $\Delta V_{\mathrm{CO}}$ & $\begin{array}{l}\text { PBL } \\
(\mathrm{m})\end{array}$ & $\begin{array}{l}\text { PA } \\
\left({ }^{\circ}\right)\end{array}$ & Baseline & $\begin{array}{c}\text { CO bandhead } \\
\text { (overtone) }\end{array}$ \\
\hline-11.47 & 9.56 & 0.82 & 0.04 & 34 & 309 & UT34 & $v=2-0$ \\
\hline-12.85 & -13.95 & 0.92 & 0.04 & 43 & 43 & UT23 & $v=2-0$ \\
\hline-13.64 & -14.01 & 0.91 & 0.03 & 44 & 45 & UT23 & $v=2-0$ \\
\hline-14.22 & -14.08 & 0.91 & 0.03 & 45 & 46 & UT23 & $v=2-0$ \\
\hline-17.29 & 9.42 & 0.76 & 0.03 & 45 & 298 & UT34 & $v=2-0$ \\
\hline-13.77 & -20.07 & 0.74 & 0.03 & 55 & 35 & UT12 & $v=2-0$ \\
\hline-14.17 & -20.20 & 0.72 & 0.03 & 56 & 36 & UT12 & $v=2-0$ \\
\hline-14.10 & -20.13 & 0.71 & 0.03 & 56 & 36 & UT12 & $v=2-0$ \\
\hline-24.32 & -4.39 & 0.81 & 0.04 & 56 & 80 & UT24 & $v=2-0$ \\
\hline-31.51 & -4.66 & 0.62 & 0.03 & 72 & 82 & UT24 & $v=2-0$ \\
\hline-26.62 & -34.02 & 0.37 & 0.03 & 98 & 39 & UT13 & $v=2-0$ \\
\hline-27.74 & -34.14 & 0.35 & 0.03 & 100 & 40 & UT13 & $v=2-0$ \\
\hline-28.39 & -34.28 & 0.35 & 0.02 & 101 & 40 & UT13 & $v=2-0$ \\
\hline-38.09 & -24.46 & 0.35 & 0.03 & 103 & 58 & UT14 & $v=2-0$ \\
\hline-45.68 & -24.86 & 0.24 & 0.03 & 119 & 62 & UT14 & $v=2-0$ \\
\hline-11.32 & 9.44 & 0.86 & 0.04 & 34 & 309 & UT34 & $v=3-1$ \\
\hline-12.69 & -13.77 & 0.95 & 0.04 & 43 & 43 & UT23 & $v=3-1$ \\
\hline-13.46 & -13.83 & 0.93 & 0.03 & 44 & 45 & UT23 & $v=3-1$ \\
\hline-14.04 & -13.90 & 0.94 & 0.03 & 45 & 46 & UT23 & $v=3-1$ \\
\hline-17.06 & 9.30 & 0.80 & 0.03 & 45 & 298 & UT34 & $v=3-1$ \\
\hline-13.59 & -19.81 & 0.78 & 0.04 & 55 & 35 & UT12 & $v=3-1$ \\
\hline-13.92 & -19.87 & 0.74 & 0.04 & 56 & 36 & UT12 & $v=3-1$ \\
\hline-13.99 & -19.94 & 0.75 & 0.04 & 56 & 36 & UT12 & $v=3-1$ \\
\hline-24.00 & -4.33 & 0.83 & 0.04 & 56 & 80 & UT24 & $v=3-1$ \\
\hline-31.10 & -4.60 & 0.69 & 0.03 & 72 & 82 & UT24 & $v=3-1$ \\
\hline-26.28 & -33.58 & 0.40 & 0.03 & 98 & 39 & UT13 & $v=3-1$ \\
\hline-27.38 & -33.70 & 0.38 & 0.03 & 100 & 40 & UT13 & $v=3-1$ \\
\hline-28.03 & -33.84 & 0.38 & 0.02 & 101 & 40 & UT13 & $v=3-1$ \\
\hline-37.60 & -24.14 & 0.37 & 0.04 & 103 & 58 & UT14 & $v=3-1$ \\
\hline-45.09 & -24.54 & 0.27 & 0.03 & 119 & 62 & UT14 & $v=3-1$ \\
\hline-11.17 & 9.32 & 0.83 & 0.03 & 34 & 309 & UT34 & $v=4-2$ \\
\hline-12.52 & -13.60 & 0.92 & 0.03 & 43 & 43 & UT23 & $v=4-2$ \\
\hline-13.29 & -13.65 & 0.88 & 0.03 & 44 & 45 & UT23 & $v=4-2$ \\
\hline-13.86 & -13.72 & 0.91 & 0.01 & 45 & 46 & UT23 & $v=4-2$ \\
\hline-16.85 & 9.18 & 0.79 & 0.03 & 45 & 298 & UT34 & $v=4-2$ \\
\hline-13.42 & -19.56 & 0.77 & 0.03 & 55 & 35 & UT12 & $v=4-2$ \\
\hline-13.74 & -19.62 & 0.71 & 0.03 & 56 & 36 & UT12 & $v=4-2$ \\
\hline-13.81 & -19.68 & 0.74 & 0.03 & 56 & 36 & UT12 & $v=4-2$ \\
\hline-23.70 & -4.28 & 0.84 & 0.03 & 56 & 80 & UT24 & $v=4-2$ \\
\hline-30.71 & -4.54 & 0.66 & 0.03 & 72 & 82 & UT24 & $v=4-2$ \\
\hline-25.94 & -33.15 & 0.39 & 0.02 & 98 & 39 & UT13 & $v=4-2$ \\
\hline-27.03 & -33.27 & 0.36 & 0.03 & 100 & 40 & UT13 & $v=4-2$ \\
\hline-27.67 & -33.40 & 0.36 & 0.01 & 101 & 40 & UT13 & $v=4-2$ \\
\hline-37.12 & -23.83 & 0.36 & 0.03 & 103 & 58 & UT14 & $v=4-2$ \\
\hline-44.51 & -24.22 & 0.27 & 0.02 & 119 & 62 & UT14 & $v=4-2$ \\
\hline-11.01 & 9.19 & 0.87 & 0.05 & 34 & 309 & UT34 & $v=5-3$ \\
\hline-12.34 & -13.40 & 0.95 & 0.06 & 43 & 43 & UT23 & $v=5-3$ \\
\hline-13.10 & -13.46 & 1.00 & 0.07 & 44 & 45 & UT23 & $v=5-3$ \\
\hline-16.60 & 9.05 & 0.84 & 0.04 & 45 & 298 & UT34 & $v=5-3$ \\
\hline-13.66 & -13.52 & 1.00 & 0.04 & 45 & 46 & UT23 & $v=5-3$ \\
\hline-13.23 & -19.28 & 0.82 & 0.05 & 55 & 35 & UT12 & $v=5-3$ \\
\hline-13.55 & -19.34 & 0.76 & 0.05 & 56 & 36 & UT12 & $v=5-3$ \\
\hline-13.61 & -19.40 & 0.80 & 0.05 & 56 & 36 & UT12 & $v=5-3$ \\
\hline-23.36 & -4.21 & 0.88 & 0.04 & 56 & 80 & UT24 & $v=5-3$ \\
\hline-30.26 & -4.48 & 0.76 & 0.04 & 72 & 82 & UT24 & $v=5-3$ \\
\hline-25.57 & -32.68 & 0.44 & 0.02 & 98 & 39 & UT13 & $v=5-3$ \\
\hline-26.64 & -32.79 & 0.42 & 0.05 & 100 & 40 & UT13 & $v=5-3$ \\
\hline-27.27 & -32.92 & 0.42 & 0.03 & 101 & 40 & UT13 & $v=5-3$ \\
\hline-36.58 & -23.49 & 0.40 & 0.03 & 103 & 58 & UT14 & $v=5-3$ \\
\hline-43.88 & -23.88 & 0.32 & 0.03 & 119 & 62 & UT14 & $v=5-3$ \\
\hline
\end{tabular}



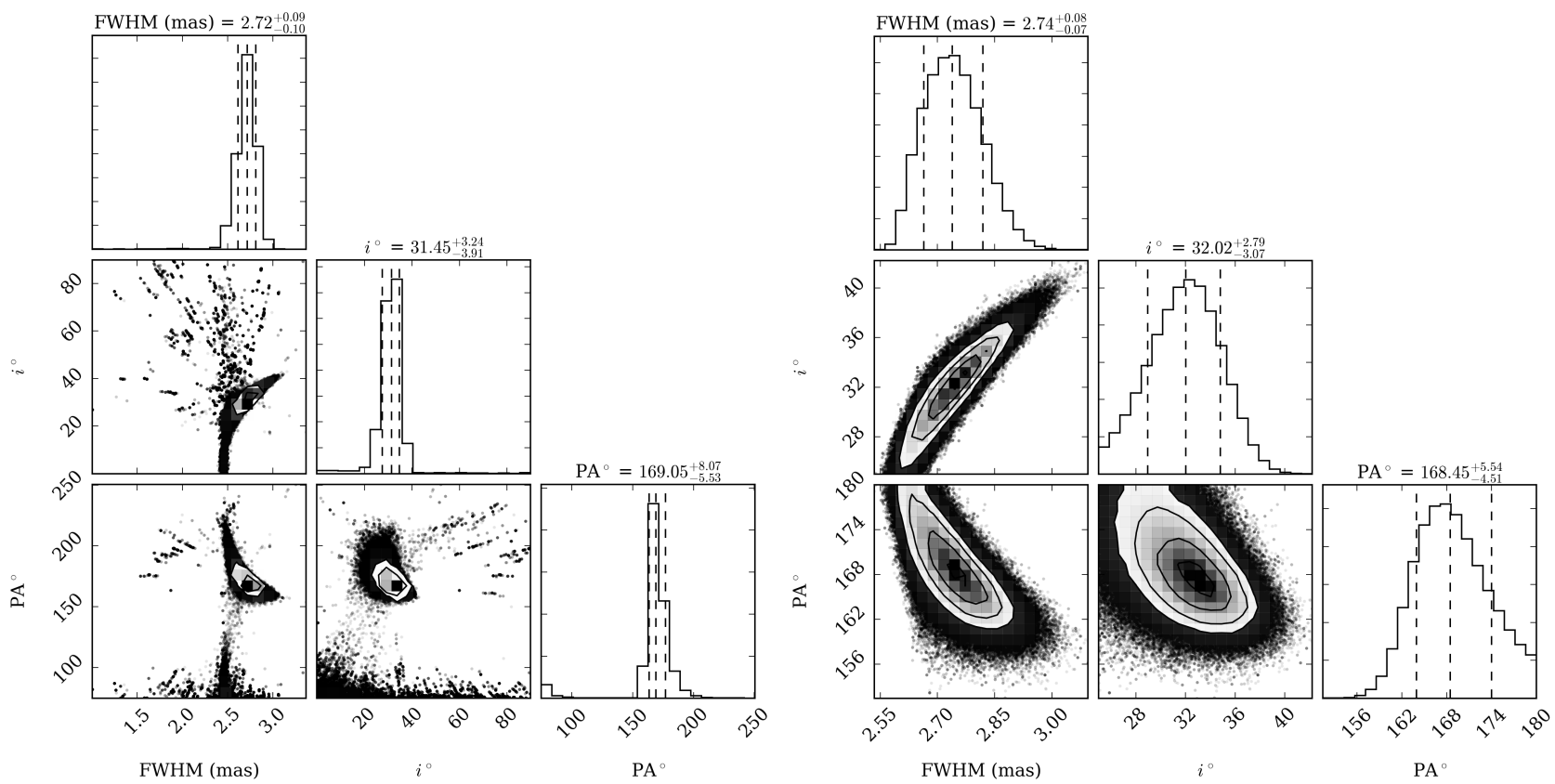

Fig. C.1. Left panel: marginal posterior distribution of the CO model for the FWHM, $i$, and PA, exploring the full range of space parameters (see text). We note that the PA varies from $75^{\circ}$ to $255^{\circ}$ for graphical reasons to keep the distribution in a single curve. The vertical dashed lines represent the 16th, 50th, and 84th percentiles reported on the top of each marginal posterior distribution plot. Right panel: same as left panel but for a more constrained range of parameters.

\section{Appendix D: Modelling results from continuum model plus $\mathrm{CO}$ geometric model}

To verify the consistency of our modelling results, we checked the visibilities obtained from our continuum model (see Sect. 3.1 and Appendix B) plus the $\mathrm{CO}$ geometrical model obtained from the pure line visibilities (see Sect. 3.2 and Appendix C) against the observed visibilities. We obtain similar results for the four observed bandheads and, as an example, Fig. D.1 shows the results for the $\mathrm{CO} v=2-0$ bandhead. Our model (black continuous line) perfectly matches the observed visibilities at the long baselines, whereas, at the short baselines, a small discrepancy (from 0.01 to 0.02 ) can be noted along the blueshifted shoulders and at peaks of the bandheads.
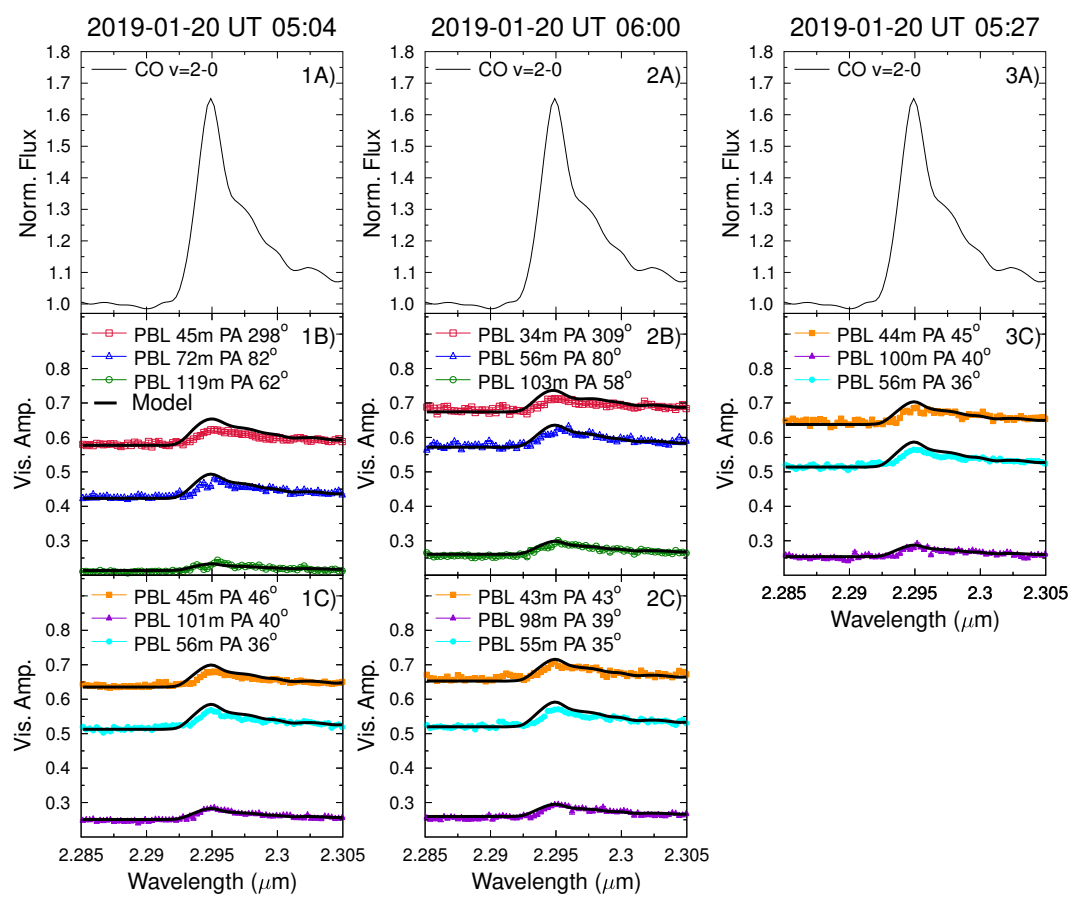

Fig. D.1. Observed visibilities (line plus continuum) of the $\mathrm{CO} v=2-0$ bandhead vs. visibilities derived from our continuum model (see Sect. 3.1 and Appendix B) plus the CO geometrical model from Eq. (C.1) (see Sect. 3.2 and Appendix C). Model visibilities for each baseline and run are overplotted as black continuous lines. 